\title{
Pseudo-Boolean Functions for Optimal Z-Complementary Code Sets with Flexible Lengths
}

\author{
Palash Sarkar, Sudhan Majhi, and Zilong Liu
}

\begin{abstract}
This paper aims to construct optimal Zcomplementary code set (ZCCS) with non-power-of-two (NPT) lengths to enable interference-free multicarrier code-division multiple access (MC-CDMA) systems. The existing ZCCSs with NPT lengths, which are constructed from generalized Boolean functions (GBFs), are sub-optimal only with respect to the set size upper bound. For the first time in the literature, we advocate the use of pseudo-Boolean functions (PBFs) (each of which transforms a number of binary variables to a real number as a natural generalization of GBF) for direct constructions of optimal ZCCSs with NPT lengths.
\end{abstract}

Index Terms-Multicarrier code-division multiple access (MCCDMA), generalized Boolean function (GBF), pseudo-Boolean function (PBF), Z-complementary code set (ZCCS), zero correlation zone $(\mathrm{ZCZ})$

\section{INTRODUCTION}

$\mathbf{M}$ ULTICARRIER code-division multiple access (MCCDMA) has been one of the most widely adopted wireless techniques in many communication systems/standards owing to its efficient fast Fourier transform (FFT) based implementation, resilience against intersymbol interference, and high spectral efficiency [1]. That being said, MC-CDMA may suffer from multiple-access interference (MAI) [2] and multipath interference (MPI) [3]. A promising way to address both MAI and MPI is to adopt proper spreading codes, such as complete complementary codes (CCC) [4] and Zcomplementary code sets (ZCCSs) [5]. This paper focuses on efficient construction of ZCCSs with a new tool, called pseudo-Boolean functions (PBFs), to enable interference-free quasi-synchronous MC-CDMA systems.

In 2007, Z-complementary pairs (ZCPs) were introduced by Fan et al. [6] to overcome the limitation on the lengths of Golay complementary pairs (GCPs) [7], [8]. A ZCP refers to a pair of sequences of same length $N$ having zero aperiodic auto-correlation sums for all time shifts $\tau$ satisfying $0<|\tau|<Z$, where $Z$ is called zero-correlation zone (ZCZ) width. When $Z=N$, the resultant sequence pair reduces to a GCP. In the literature, there are direct constructions of GCPs and ZCPs with the aid of generalized Boolean functions (GBFs) [9]-[11]. The idea of ZCPs introduced in [6] was generalized to ZCCS by Feng et al. in [12]. A ZCCS refers to a set of $K$ codes, each of which consists of $M$ constituent sequences of identical length $L$, having ideal aperiodic autoand cross-correlation properties inside the ZCZ width and

Palash Sarkar and Sudhan Majhi are with the Department of EE, IIT Patna, India, e-mail: \{ palash.pma15,smajhi\}@iitp.ac.in.

Zilong Liu is with the School of CSEE, University of Essex, UK, email:zilong.liudessex.ac.uk.
TABLE I

COMPARISON OF THE Proposed CONSTRUCTION With [5], [21]-[24], [27]

\begin{tabular}{|l|l|l|l|l|l|}
\hline ZCCS & Method & Length $(N)$ & $\left\lfloor\frac{N}{Z}\right\rfloor$ & Constraints & Optimality \\
\hline$[5]$ & Direct & $2^{m}$ & $\geq 2$ & $m \geq 2$ & Optimal \\
\hline$[23 \mid$ & Direct & $2^{m}$ & $\geq 2$ & $m \geq 2$ & Optimal \\
\hline$[24 \mid$ & Direct & $2^{m}+2$ & $=1$ & $m \geq 4$ & Sub-optimal \\
\hline$[21 \mid$ & Direct & $2^{m}$ & $\geq 2$ & $m \geq 2$ & Optimal \\
\hline$[22 \mid$ & Direct & $2^{m}$ & $\geq 2$ & $m \geq 2$ & Optimal \\
\hline$[22 \mid$ & Direct & $2^{m}+2^{h}$ & $\geq 1$ & $m>0,0<h \leq m$ & Non-optimal \\
\hline$[27 \mid$ & Indirect & $L$ & $\geq 2$ & $L \geq 1$ & Optimal \\
\hline Theorem 1 & Direct & $p 2^{m}$ & $\geq 2$ & $m \geq 2$, prime $p$ & Optimal \\
\hline
\end{tabular}

satisfying the theoretical upper bound: $K \leq M\lfloor N / Z\rfloor$ [13]. When $Z=N$, the set is called a mutually orthogonal Golay complementary sets (MOGCSs) [4], which refers to collection of complementary codes (CCs) [14]-[16] with ideal crosscorrelation properties. A set of CCCs is known as a MOGCSs with the equality $K=M$ [17]-[20]. The theoretical upper bound shows that an optimal ZCCS has larger set size as compared to CCCs provided $\left\lfloor\frac{N}{Z}\right\rfloor \geq 2$. Recently, several GBFs based constructions of optimal ZCCSs with powerof-two lengths have been reported in [5], [21]-[23]. In the recent literature, two direct constructions of ZCCSs with NPT lengths can be found in [24] and [22], which produces suboptimal ZCCS with $\left\lfloor\frac{N}{Z}\right\rfloor=1$ and non-optimal ZCCSs for NPT lengths with $\left\lfloor\frac{N}{Z}\right\rfloor<1$, respectively. To the best of our knowledge, the construction of optimal ZCCSs of NPT lengths with $\left\lfloor\frac{N}{Z}\right\rfloor \geq 2$, based on GBFs remains open. Other methods which are dependent on the existence of special sequences, known as indirect constructions [11], to construct ZCCSs can be found in [25]-[27]. The indirect constructions heavily rely on a series of sequence operations which may not be feasible for rapid hardware generation, especially, when the sequence lengths are large [5].

It is noted that the MAI in MC-CDMA system can be mitigated using zero-correlation properties of a ZCCS provided that all the received multiuser signals are roughly synchronous within the ZCZ width [19]. In addition to their applications in MC-CDMA [18], [19], [27], ZCCSs have also been employed as optimal training sequences in multiple-input multiple-output (MIMO) communications [28], [29]. The limitation on the set size of CCCs and the unavailability of optimal ZCCSs with NPT lengths using direct constructions in the existing literature are a major motivation of this work. Specifically, for the first time in the literature, we propose to use PBFs for direct construction of optimal ZCCS of lengths $p 2^{m}$, where $p$ is a prime number and $m$ is a positive integer. A PBF [30] refers to an arbitrary mapping of the set of binary $m$-tuples to 
real numbers. Being a natural generalization of GBFs, PBFs are also suitable for rapid hardware generation of sequences. A detailed comparison of the proposed construction with [5], [21]-[24], [27] is given in TABLE I.

\section{PRELIMINARY}

In this section, we present some basic definitions and lemmas to be used in the proposed construction. Let $\mathbf{y}_{1}=$ $\left(y_{1,0}, y_{1,1}, \cdots, y_{1, N-1}\right)$ and $\mathbf{y}_{2}=\left(y_{2,0}, y_{2,1}, \cdots, y_{2, N-1}\right)$ denote a pair of sequences with complex components. For an integer $\tau$, define [5]

$$
\theta\left(\mathbf{y}_{1}, \mathbf{y}_{2}\right)(\tau)= \begin{cases}\sum_{i=0}^{N-1-\tau} y_{1, i+\tau} y_{2, i}^{*}, & 0 \leq \tau<N, \\ \sum_{i=0}^{N+\tau-1} y_{1, i} y_{2, i-\tau}^{*}, & -N<\tau<0, \\ 0, & \text { otherwise, }\end{cases}
$$

The functions $\theta\left(\mathbf{y}_{1}, \mathbf{y}_{2}\right)$ and $\theta\left(\mathbf{y}_{1}, \mathbf{y}_{1}\right)$ are called the aperiodic cross-correlation function (ACCF) between $\mathbf{y}_{1}$ and $\mathbf{y}_{2}$, and the aperiodic auto-correlation function (AACF) of $\mathbf{y}_{1}$, respectively. Let $\mathcal{S}=\left\{\mathcal{S}_{0}, \mathcal{S}_{1}, \cdots, \mathcal{S}_{K-1}\right\}$ be a set of $K$ codes or ordered sets defined as

$$
\mathcal{S}_{\mu}=\left(\mathbf{s}_{0}^{\mu}, \mathbf{s}_{1}^{\mu}, \ldots, \mathbf{s}_{M-1}^{\mu}\right) \text {, }
$$

where $\mathbf{s}_{\nu}^{\mu}(0 \leq \nu \leq M-1,0 \leq \mu \leq K-1)$ is the $\nu$-th element which we assume is a complex-valued sequence of length $N$ in $\mathcal{S}_{\mu}$. For $\mathcal{S}_{\mu_{1}}, \mathcal{S}_{\mu_{2}} \in \mathcal{S}\left(0 \leq \mu_{1}, \mu_{2} \leq K-1\right)$, the ACCF between $\mathcal{S}_{\mu_{1}}$ and $\mathcal{S}_{\mu_{2}}$ is defined as

$$
\theta\left(\mathcal{S}_{\mu_{1}}, \mathcal{S}_{\mu_{2}}\right)(\tau)=\sum_{\nu=0}^{M-1} \theta\left(\mathbf{s}_{\nu}^{\mu_{1}}, \mathbf{s}_{\nu}^{\mu_{2}}\right)(\tau)
$$

Definition 1 ( $[5] /)$ : Code set $\mathcal{S}$ is called a ZCCS if

$$
\theta\left(\mathcal{S}_{\mu_{1}}, \mathcal{S}_{\mu_{2}}\right)(\tau)= \begin{cases}M N, & \tau=0, \mu_{1}=\mu_{2}, \\ 0, & 0<|\tau|<Z, \mu_{1}=\mu_{2}, \\ 0, & |\tau|<Z, \mu_{1} \neq \mu_{2},\end{cases}
$$

where $Z$ is called ZCZ width. We denote a ZCCS with the parameters $K, N, M$, and $Z$ by the notation $(K, Z)-\operatorname{ZCCS}_{M}^{N}$. For $K=M$ and $Z=N$, a $(K, Z)-Z_{C C S}^{N}$ is called a set of CCCs and we denote it by $(K, K, N)$-CCC.

We call a $(K, Z)-Z_{C C S}^{N}$ optimal if it achieves the equality in the theoretical upper-bound, given by $K \leq M\left\lfloor\frac{N}{Z}\right\rfloor[13]$.

\section{A. Generalized Boolean Functions (GBFs)}

Let $x_{0}, x_{1}, \ldots, x_{m-1}$ denote $m$ variables which take values from $\mathbb{Z}_{2}$. A monomial of degree $i(0 \leq i \leq m)$ is defined as the product of any $i$ distinct variables among $x_{0}, x_{1}, \ldots, x_{m-1}$. Let us assume that $\mathcal{A}_{i}$ denotes the set of all monomials of degree $i$, where

$$
\begin{aligned}
\mathcal{A}_{i}= & \left\{x_{0}^{r_{0}} x_{1}^{r_{1}} \cdots x_{m-1}^{r_{m-1}}:\right. \\
& \left.r_{0}+r_{1}+\cdots+r_{m-1}=i,\left(r_{0}, r_{1}, \ldots, r_{m-1}\right) \in \mathbb{Z}_{2}^{m}\right\} .
\end{aligned}
$$

A function $f: \mathbb{Z}_{2}^{m} \rightarrow \mathbb{Z}_{q}$ is said to be a GBF if it can uniquely be expressed as a linear combination of the monomials in $\mathcal{A}_{m}$, where the coefficient of each monomial is drawn from $\mathbb{Z}_{q}$, where $\mathbb{Z}_{q}$ denotes the set of integers modulo $q$. The highest degree monomial with non-zero coefficient present in the expression of $f$ determine the order of $f$. As an example, $2 x_{0} x_{1}+x_{1}+1$ is a second order GBF of two variables $x_{0}$ and $x_{1}$ over $\mathbb{Z}_{3}$. We denote the graph of a secondorder GBF $f$ by $G(f)$ [14]. It contains $m$ vertices which are denoted by the $m$ variables of $f$. The edges in the $G(f)$ are determined by the second-degree monomials present in the expression of $f$ with non-zero coefficients. There is an edge of weight $w$ between the vertices $x_{\alpha}$ and $x_{\beta}$ of $G(f)$ if the expression of $f$ contains the term $w x_{\alpha} x_{\beta}$. Let $\psi(f)$ denotes the complex-valued sequence corresponding to a GBF $f$ and it is defined as [14], $\psi(f)=\left(\omega_{q}^{f_{0}}, \omega_{q}^{f_{1}}, \ldots, \omega_{q}^{f_{2} m_{-1}}\right)$, where $\omega_{q}$ denotes $\exp (2 \pi \sqrt{-1} / q), f_{r}=f\left(r_{0}, r_{1}, \ldots, r_{m-1}\right)$, $\left(r_{0}, r_{1}, \ldots, r_{m-1}\right)$ is the binary vector representation of integer $r\left(r=\sum_{\alpha=0}^{m-1} r_{\alpha} 2^{\alpha}\right)$, and $q$ denotes an even number, no less than 2 . We denote by $\bar{x}=1-x$ the binary complement of $x \in\{0,1\}$. For any given GBF $f$ in $m$ variables, we denote the function $f\left(1-x_{0}, 1-x_{1}, \ldots, 1-x_{m-1}\right)$ or $f\left(\bar{x}_{0}, \bar{x}_{1}, \ldots, \bar{x}_{m-1}\right)$ by $\tilde{f}$. Let $\mathcal{C}=\left(g_{1}, g_{2}, \ldots, g_{M}\right)$ be an ordered set of $M$ GBFs. We define the code $\psi(\mathcal{C})$ corresponding to $\mathcal{C}$ as $\psi(\mathcal{C})=\left(\psi\left(g_{1}\right), \psi\left(g_{2}\right), \ldots, \psi\left(g_{M}\right)\right)$.

Lemma 1: (Construction of CCC [4])

Let $f: \mathbb{Z}_{2}^{m} \rightarrow \mathbb{Z}_{q}$ be a second-order GBF. Let us assume that $G(f)$ contains the vertices $x_{j_{0}}, x_{j_{1}}, \ldots, x_{j_{k-1}}$ such a way that after performing a deletion operation on those vertices, the resulting graph reduces to a path. Let the edges in the path have identical weight of $\frac{q}{2}$ and $\mathbf{t}=\left(t_{0}, t_{1}, \cdots, t_{k-1}\right)$ be the binary representation of the integer $t$. Define the $\mathrm{CC}, C_{t}$ to be

$$
\left\{f+\frac{q}{2}\left((\mathbf{d}+\mathbf{t}) \cdot \mathbf{x}+d x_{\gamma}\right): \mathbf{d} \in\{0,1\}^{k}, d \in\{0,1\}\right\},
$$

and $\bar{C}_{t}$ to be

$$
\left\{\tilde{f}+\frac{q}{2}\left((\mathbf{d}+\mathbf{t}) \cdot \overline{\mathbf{x}}+\bar{d} x_{\gamma}\right): \mathbf{d} \in\{0,1\}^{k}, d \in\{0,1\}\right\},
$$

where $(\cdot) \cdot(\cdot)$ denotes the dot product between two real-valued vector $(\cdot)$ and $(\cdot), \gamma$ is the label of either end vertex in the path, $\mathbf{x}=\left(x_{j_{0}}, x_{j_{1}}, \ldots, x_{j_{k-1}}\right), \overline{\mathbf{x}}=\left(1-x_{j_{0}}, 1-x_{j_{1}}, \ldots, 1-\right.$ $\left.x_{j_{k-1}}\right)$, and $\mathbf{d}=\left(d_{0}, d_{1}, \ldots, d_{k-1}\right)$. Then $\left\{\psi\left(C_{t}\right), \psi^{*}\left(\bar{C}_{t}\right)\right.$ : $\left.0 \leq t<2^{k}\right\}$ forms $\left(2^{k+1}, 2^{k+1}, 2^{m}\right)$-CCC, where $\psi^{*}(\cdot)$ denotes the complex conjugate of $\psi(\cdot)$.

\section{B. Pseudo-Boolean Functions (PBFs)}

A function $F:\{0,1\}^{m} \rightarrow \mathbb{R}$ is said to be a PBF if it can be uniquely expressed as a linear combination of monomials in $\mathcal{A}_{m}$ with the coefficients drawn from $\mathbb{R}$, where $\mathbb{R}$ denotes the set of real numbers. Therefore, PBFs are a natural generalization of GBFs [30]. As an example, $\frac{2}{3} x_{0} x_{1}+x_{0}+1$ is a second-order PBF of two variables $x_{0}$ and $x_{1}$ but not a GBF. Let $f: \mathbb{Z}_{2}^{m} \rightarrow \mathbb{Z}_{q}$ be a GBF of the variables $x_{0}, x_{1}, \ldots, x_{m-1}$. Let us assume that $p$ denotes a prime number and define the following PBFs with the help of the GBF $f$ :

$$
\begin{aligned}
F^{\lambda} & =f+\frac{\lambda q}{p}\left(x_{m}+2 x_{m+1}+\cdots+2^{s-1} x_{m+s-1}\right), \\
G^{\lambda} & =\tilde{f}+\frac{\lambda q}{p}\left(x_{m}+2 x_{m+1}+\cdots+2^{s-1} x_{m+s-1}\right),
\end{aligned}
$$


where $s \in \mathbb{Z}^{+}$which denotes the set of all positive integers, $2 \leq p<2^{s+1}$, and $\lambda=0,1, \ldots, p-1$. From (8), it is clear that $F^{\lambda}$ and $G^{\lambda}$ are PBFs of $m+s$ variables $x_{0}, x_{1}, \ldots, x_{m+s-1}$. From (8), it can be observed that the PBFs $F^{\lambda}$ and $G^{\lambda}$ reduce to $\mathbb{Z}_{q}$-valued GBFs if $p$ divides $q$.

\section{Proposed Construction of Z-Complementary Code SET}

In this section, we shall present our proposed construction of ZCCS using PBFs. To this end, we first present a lemma which will be used in our proposed construction.

Lemma 2: ( [31]) Let $\lambda$ and $\lambda^{\prime}$ be two non-negative integers, where $0 \leq \lambda \neq \lambda^{\prime}<p, p$ is a prime number as defined in Section-II. Then $\sum_{\alpha=0}^{p-1} \omega_{p}^{\left(\lambda-\lambda^{\prime}\right) \alpha}=0$.

For $0 \leq t<2^{k}$ and $0 \leq \lambda<p$, we define the following sets of PBFs:

$$
U_{t}^{\lambda}=\left\{F^{\lambda}+\frac{q}{2}\left((\mathbf{d}+\mathbf{t}) \cdot \mathbf{x}+d x_{\gamma}\right): \mathbf{d} \in\{0,1\}^{k}, d \in\{0,1\}\right\},
$$

and

$$
V_{t}^{\lambda}=\left\{G^{\lambda}+\frac{q}{2}\left((\mathbf{d}+\mathbf{t}) \cdot \overline{\mathbf{x}}+\bar{d} x_{\gamma}\right): \mathbf{d} \in\{0,1\}^{k}, d \in\{0,1\}\right\} .
$$

Let us assume that $f^{\mathbf{d}, \mathbf{t}, d}=f+\frac{q}{2}\left((\mathbf{d}+\mathbf{t}) \cdot \mathbf{x}+d x_{\gamma}\right), g^{\mathbf{d}, \mathbf{t}, d}=$ $\tilde{f}+\frac{q}{2}\left((\mathbf{d}+\mathbf{t}) \cdot \overline{\mathbf{x}}+\bar{d} x_{\gamma}\right)$, in Lemma 1 We also assume $F^{\mathbf{d}, \mathbf{t}, d, \lambda}=$ $F^{\lambda}+\frac{q}{2}((\mathbf{d}+\mathbf{t}) \cdot \mathbf{x})+d x_{\gamma}$, in (9), and $G^{\mathbf{d}, \mathbf{t}, d, \lambda}=G^{\lambda}+\frac{q}{2}((\mathbf{d}+$ $\left.\mathbf{t}) \cdot \overline{\mathbf{x}}+\bar{d} x_{\gamma}\right)$, in (10). As per our assumption, for any choice of $\mathbf{d}, \mathbf{t} \in\{0,1\}^{k}$, and $d \in\{0,1\}$, the functions $f^{\mathbf{d}, \mathbf{t}, d}$ and $g^{\mathbf{d}, \mathbf{t}, d}$ are $\mathbb{Z}_{q}$-valued GBFs of $m$ variables. For any choice of $\mathbf{d}, \mathbf{t} \in$ $\{0,1\}^{k}, d \in\{0,1\}$, and $\lambda \in\{0,1, \ldots, p-1\}$, the functions $F^{\mathbf{d}, \mathbf{t}, d, \lambda}$ and $G^{\mathbf{d}, \mathbf{t}, d, \lambda}$ are PBFs of $m+s$ variables. We define $\psi\left(F^{\mathbf{d}, \mathbf{t}, d, \lambda}\right)$, the complex-valued sequence corresponding to $F^{\mathbf{d}, \mathbf{t}, d, \lambda}$, as

$$
\psi\left(F^{\mathbf{d}, \mathbf{t}, d, \lambda}\right)=\left(\omega_{q}^{F_{0}^{\mathbf{d}, \mathbf{t}, d, \lambda}}, \omega_{q}^{F_{1}^{\mathbf{d}, \mathbf{t}, d, \lambda}}, \ldots, \omega_{q}^{F^{\mathbf{d}, \mathbf{t}, d, \lambda}-1}\right),
$$

where $F_{r^{\prime}}^{\mathbf{d}, \mathbf{t}, d, \lambda}=F^{\mathbf{d}, \mathbf{t}, d, \lambda}\left(r_{0}, r_{1}, \cdots, r_{m+s-1}\right), r^{\prime}=$ $\sum_{\alpha=0}^{m+s-1} r_{\alpha} 2^{\alpha}$. The $r^{\prime}$-th component of $\psi\left(F^{\mathbf{d}, \mathbf{t}, d, \lambda}\right)$ is given by

$$
\begin{aligned}
\omega_{q}^{F^{\mathbf{d}, \mathbf{t}, d, \lambda}} & =\omega_{q}^{f^{\mathbf{d}, \mathbf{t}, d}\left(r_{0}, r_{1}, \ldots, r_{m-1}\right)+\frac{q \lambda}{p}\left(r_{m}+2 r_{m+1}+\cdots+2^{s-1} r_{m+s-1}\right)} \\
& =\omega_{q}^{f_{r}^{\mathbf{d}, \mathbf{t}, d}} \omega_{p}^{\lambda\left(r_{m}+2 r_{m+1}+\cdots+2^{s-1} r_{m+s-1}\right)} .
\end{aligned}
$$

From (12), it can be observed that $\omega_{q}^{F_{r^{\prime}}^{\mathrm{d}, \mathrm{t}, d, \lambda}}$ is a root of the polynomial: $z^{\delta}-1$, where $\delta=\operatorname{lcm}(p, q)$, denotes a positive integer given by the least common multiple $(\mathrm{lcm})$ of $p$ and $q$. Therefore, the components of $\psi\left(F^{\mathbf{d}, \mathbf{t}, d, \lambda}\right)$ are given by the roots of the polynomial: $z^{\delta}-1$. From (11) and 12), we have

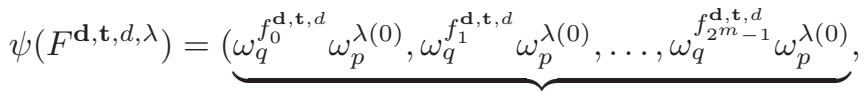

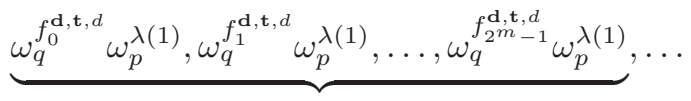

$$
\begin{aligned}
& \underbrace{\left.\omega_{q}^{f_{0}^{\mathbf{d}, \mathbf{t}, d}} \omega_{p}^{\lambda\left(2^{s}-1\right)}, \omega_{q}^{f_{1}^{\mathbf{d}, \mathbf{t}, d}} \omega_{p}^{\lambda\left(2^{s}-1\right)}, \ldots, \omega_{q}^{f_{2}^{\mathbf{d}, \mathbf{t}, d}-1} \omega_{p}^{\lambda\left(2^{s}-1\right)}\right)} .
\end{aligned}
$$

Let us also define $\psi_{2^{m+s}-p 2^{m}}\left(F^{\mathbf{d}, \mathbf{t}, d, \lambda}\right)$ which is defined to be obtained from $\psi\left(F^{\mathbf{d}, \mathbf{t}, d, \lambda}\right)$ by removing its last $2^{m+s}-p 2^{m}$ components.

$$
\begin{aligned}
& \psi_{2^{m+s}-p 2^{m}}\left(F^{\mathbf{d}, \mathbf{t}, d, \lambda}\right)
\end{aligned}
$$

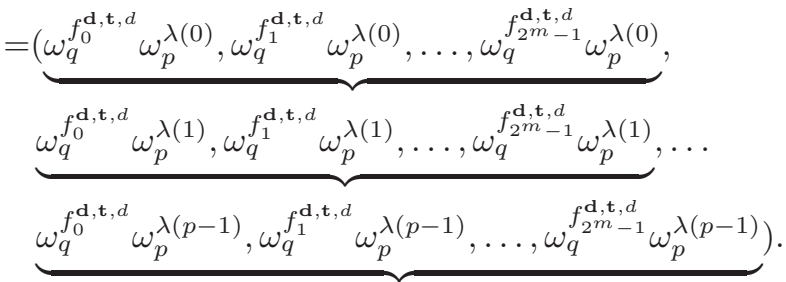

Similarly, we can also obtain $\psi_{2^{m+s}-p 2^{m}}\left(G^{\mathbf{d}, \mathbf{t}, d, \lambda}\right)$ as

$$
\begin{aligned}
& \psi_{2^{m+s}-p 2^{m}}\left(G^{\mathbf{d}, \mathbf{t}, d, \lambda}\right) \\
& =\underbrace{\omega_{q}^{g_{0}^{\mathbf{d}, \mathbf{t}, d}} \omega_{p}^{\lambda(0)}, \omega_{q}^{g_{1}^{\mathbf{d}, \mathbf{t}, d}} \omega_{p}^{\lambda(0)}, \ldots, \omega_{q}^{g_{2}^{\mathbf{d}, \mathbf{t}, d}}{ }^{-1} \omega_{p}^{\lambda(0)}}, \\
& \underbrace{\omega_{q}^{g_{0}^{\mathbf{d}, \mathbf{t}, d}} \omega_{p}^{\lambda(1)}, \omega_{q}^{g_{1}^{\mathbf{d}, \mathbf{t}, d}} \omega_{p}^{\lambda(1)}, \ldots, \omega_{q}^{g_{2}^{\mathbf{d}, \mathbf{t}, d}-1} \omega_{p}^{\lambda(1)}}, \ldots \\
& \underbrace{\left.\omega_{q}^{g_{0}^{\mathbf{d}, \mathbf{t}, d}} \omega_{p}^{\lambda(p-1)}, \omega_{q}^{g_{1}^{\mathbf{d}, \mathbf{t}, d}} \omega_{p}^{\lambda(p-1)}, \ldots, \omega_{q}^{g_{2}^{\mathbf{d}, \mathbf{t}, d}} \omega_{p}^{\lambda(p-1)}\right)} .
\end{aligned}
$$

Theorem 1: Let $f: \mathbb{Z}_{2}^{m} \rightarrow \mathbb{Z}_{q}^{m}$ be a GBF as defined in Lemma 11. Then the set of codes

$$
\left\{\psi_{2^{m+s}-p 2^{m}}\left(U_{t}^{\lambda}\right), \psi_{2^{m+s}-p 2^{m}}^{*}\left(V_{t}^{\lambda}\right): 0 \leq t<2^{k}, 0 \leq \lambda<p\right\},
$$

forms $\left(p 2^{k+1}, 2^{m}\right)-\mathrm{ZCCS}_{2^{k+1}}^{p 2^{m}}$.

Proof: In (13), 14), and (15), each of the parentheses below a complex-valued sequence contains $2^{m}$ components of the complex-valued sequence. It can be observed that the $2^{m}$ components in the $i$-th parentheses of $\psi_{2^{m+s}-p 2^{m}}\left(F^{\mathbf{d}, \mathbf{t}, d, \lambda}\right)$ and $\psi_{2^{m+s}-p 2^{m}}\left(G^{\mathbf{d}, \mathbf{t}, d, \lambda}\right)$ represent the complex-valued sequences $\omega_{p}^{\lambda(i-1)} \psi\left(f^{\mathbf{d}, \mathbf{t}}\right)$ and $\omega_{p}^{\lambda(i-1)} \psi\left(g^{\mathbf{d}, \mathbf{t}}\right)$, respectively, where $i=1,2, \ldots, p$. Using (9), (14), Lemma 1 and Lemma 2 the ACCF between $\psi_{2^{m+s}-p 2^{m}}\left(U_{t}^{\lambda}\right)$ and $\psi_{2^{m+s}-p 2^{m}}\left(U_{t^{\prime}}^{\lambda^{\prime}}\right)$ for $\tau=0$ can be derived as follows:

$$
\begin{aligned}
& \theta\left(\psi_{2^{m+s}-p 2^{m}}\left(U_{t}^{\lambda}\right), \psi_{2^{m+s}-p 2^{m}}\left(U_{t^{\prime}}^{\lambda^{\prime}}\right)\right)(0) \\
& =\sum_{\mathbf{d}, d} \theta\left(\psi_{2^{m+s}-p 2^{m}}\left(F^{\mathbf{d}, \mathbf{t}, d, \lambda}\right), \psi_{2^{m+s}-p 2^{m}}\left(F^{\mathbf{d}, \mathbf{t}^{\prime}, d, \lambda^{\prime}}\right)\right)(0) \\
& =\sum_{\mathbf{d}, d} \theta\left(\psi\left(f^{\mathbf{d}, \mathbf{t}, d}\right), \psi\left(f^{\mathbf{d}, \mathbf{t}^{\prime}, d}\right)\right)(0) \sum_{\alpha=0}^{p-1} \omega_{p}^{\left(\lambda-\lambda^{\prime}\right) \alpha} \\
& =\theta\left(\psi\left(C_{t}\right), \psi\left(C_{t^{\prime}}\right)\right)(0) \sum_{\alpha=0}^{p-1} \omega_{p}^{\left(\lambda-\lambda^{\prime}\right) \alpha} \\
& = \begin{cases}p 2^{m+k+1}, & t=t^{\prime}, \lambda=\lambda^{\prime}, \\
0, & t=t^{\prime}, \lambda \neq \lambda^{\prime}, \\
0, & t \neq t^{\prime}, \lambda=\lambda^{\prime}, \\
0, & t \neq t^{\prime}, \lambda \neq \lambda^{\prime} .\end{cases}
\end{aligned}
$$


Again, Using (9), (14), and Lemma 17 the ACCF between $\psi_{2^{m+s}-p 2^{m}}\left(U_{t}^{\lambda}\right)$ and $\psi_{2^{m+s}-p 2^{m}}\left(U_{t^{\prime}}^{\lambda^{\prime}}\right)$ for $0<|\tau|<2^{m}$ can be derived as

$$
\begin{aligned}
& \theta\left(\psi_{2^{m+s}-p 2^{m}}\left(U_{t}^{\lambda}\right), \psi_{2^{m+s}-p 2^{m}}\left(U_{t^{\prime}}^{\lambda^{\prime}}\right)\right)(\tau) \\
= & \theta\left(\psi\left(C_{t}\right), \psi\left(C_{t^{\prime}}\right)\right)(\tau) \sum_{\alpha=0}^{p-1} \omega_{p}^{\left(\lambda-\lambda^{\prime}\right) \alpha} \\
& +\theta\left(\psi\left(C_{t}\right), \psi\left(C_{t^{\prime}}\right)\right)\left(\tau-2^{m}\right) \sum_{\alpha=0}^{p-2} \omega_{p}^{\lambda(\alpha+1)-\lambda^{\prime} \alpha} .
\end{aligned}
$$

From Lemma 7 we have

$$
\theta\left(\psi\left(C_{t}\right), \psi\left(C_{t^{\prime}}\right)\right)(\tau)=0,0<|\tau|<2^{m} .
$$

From (18) and 199, we have

$$
\theta\left(\psi_{2^{m+s}-p 2^{m}}\left(U_{t}^{\lambda}\right), \psi_{2^{m+s}-p 2^{m}}\left(U_{t^{\prime}}^{\lambda^{\prime}}\right)\right)(\tau)=0,0<|\tau|<2^{m} .
$$

From (17) and 20), we have

$$
\begin{aligned}
& \theta\left(\psi_{2^{m+s}-p 2^{m}}\left(U_{t}^{\lambda}\right), \psi_{2^{m+s}-p 2^{m}}\left(U_{t^{\prime}}^{\lambda^{\prime}}\right)\right)(\tau) \\
& \quad= \begin{cases}p 2^{m+k+1}, & t=t^{\prime}, \lambda=\lambda^{\prime}, \tau=0, \\
0, & t=t^{\prime}, \lambda \neq \lambda^{\prime}, 0<|\tau|<2^{m}, \\
0, & t \neq t^{\prime}, \lambda=\lambda^{\prime}, 0<|\tau|<2^{m}, \\
0, & t \neq t^{\prime}, \lambda \neq \lambda^{\prime}, 0<|\tau|<2^{m} .\end{cases}
\end{aligned}
$$

Similarly, it can be shown that

$$
\begin{aligned}
& \theta\left(\psi_{2^{m+s}-p 2^{m}}^{*}\left(V_{t}^{\lambda}\right), \psi_{2^{m+s}-p 2^{m}}^{*}\left(V_{t^{\prime}}^{\lambda^{\prime}}\right)\right)(\tau) \\
& = \begin{cases}p 2^{m+k+1}, & t=t^{\prime}, \lambda=\lambda^{\prime}, \tau=0, \\
0, & t=t^{\prime}, \lambda \neq \lambda^{\prime}, 0<|\tau|<2^{m}, \\
0, & t \neq t^{\prime}, \lambda=\lambda^{\prime}, 0<|\tau|<2^{m}, \\
0, & t \neq t^{\prime}, \lambda \neq \lambda^{\prime}, 0<|\tau|<2^{m} .\end{cases}
\end{aligned}
$$

From Lemma (1, (9), 10), (14), and (15), the ACCF between $\psi_{2^{m+s}-p 2^{m}}\left(U_{t}^{\lambda}\right)$ and $\psi_{2^{m+s}-p 2^{m}}^{*}\left(V_{t^{\prime}}^{\lambda^{\prime}}\right)$ for $\tau=0$ can be derived as

$$
\begin{gathered}
\theta\left(\psi_{2^{m+s}-p 2^{m}}\left(U_{t}^{\lambda}\right), \psi_{2^{m+s}-p 2^{m}}^{*}\left(V_{t^{\prime}}^{\lambda^{\prime}}\right)\right)(0) \\
\quad=\theta\left(\psi\left(C_{t}\right), \psi^{*}\left(\bar{C}_{t^{\prime}}\right)\right)(0) \sum_{\alpha=0}^{p-1} \omega_{p}^{\left(\lambda+\lambda^{\prime}\right) \alpha}
\end{gathered}
$$

From Lemma $\square$ we have

$$
\theta\left(\psi\left(C_{t}\right), \psi^{*}\left(\bar{C}_{t^{\prime}}\right)\right)(0)=0 .
$$

From 23) and 24, we have

$$
\theta\left(\psi_{2^{m+s}-p 2^{m}}\left(U_{t}^{\lambda}\right), \psi_{2^{m+s}-p 2^{m}}^{*}\left(V_{t^{\prime}}^{\lambda^{\prime}}\right)\right)(0)=0 .
$$

From Lemma 1 (9), (10), (14), (15), and (24), the ACCF between $\psi_{2^{m+s}-p 2^{m}}\left(U_{t}^{\lambda}\right)$ and $\psi_{2^{m+s}-p 2^{m}}^{*}\left(V_{t^{\prime}}^{\lambda^{\prime}}\right)$ for $0<|\tau|<$ $2^{m}$ can be derived as

$$
\begin{aligned}
& \theta\left(\psi_{2^{m+s}-p 2^{m}}\left(U_{t}^{\lambda}\right), \psi_{2^{m+s}-p 2^{m}}^{*}\left(V_{t^{\prime}}^{\lambda^{\prime}}\right)\right)(\tau) \\
= & \theta\left(\psi\left(C_{t}\right), \psi^{*}\left(\bar{C}_{t^{\prime}}\right)\right)(\tau) \sum_{\alpha=0}^{p-1} \omega_{p}^{\left(\lambda+\lambda^{\prime}\right) \alpha} \\
& +\theta\left(\psi\left(C_{t}\right), \psi^{*}\left(\bar{C}_{t^{\prime}}\right)\right)\left(\tau-2^{m}\right) \sum_{\alpha=0}^{p-2} \omega_{p}^{\lambda(\alpha+1)+\lambda^{\prime} \alpha}
\end{aligned}
$$

$=0$.
From (25) and 26, we have

$$
\theta\left(\psi_{2^{m+s}-p 2^{m}}\left(U_{t}^{\lambda}\right), \psi_{2^{m+s}-p 2^{m}}^{*}\left(V_{t^{\prime}}^{\lambda^{\prime}}\right)\right)(\tau)=0,|\tau|<2^{m} .
$$

The obtained results in 201, 227, and 27) show that the following set of codes

$\left\{\psi_{2^{m+s}-p 2^{m}}\left(U_{t}^{\lambda}\right), \psi_{2^{m+s}-p 2^{m}}^{*}\left(V_{t}^{\lambda}\right): 0 \leq t<2^{k}, 0 \leq \lambda<p\right\}$

forms $\left(p 2^{k+1}, 2^{m}\right)-\mathrm{ZCCS}_{2^{k+1}}^{p^{m}}$.

The proposed $\left(p 2^{k+1}, 2^{m}\right)-\mathrm{ZCCS}_{2^{k+1}}^{p 2^{m}}$ is optimal as it satisfies the equality $K=M\left\lfloor\frac{N}{Z}\right\rfloor$.

Remark 1: For $p=2, \delta=l c m(p, q)=q$, and the PBFs $F^{\lambda}$ and $G^{\lambda}$ become GBFs of $m+s$ variables over $\mathbb{Z}_{q}$. For the same value of $p$, from Theorem 1, we obtain $\left(2^{k+2}, 2^{m}\right)-\mathrm{ZCCS}_{2^{k+1}}^{2^{m+1}}$ which is optimal and the components of each codewords from a code in $\left(2^{k+2}, 2^{m}\right)-\mathrm{ZCCS}_{2^{2+1}}^{2^{m+1}}$ are drawn from the roots of the polynomial: $z^{q}-1$. Therefore, the proposed construction also generates ZCCSs of length in the form of power-of-two over the ring $\mathbb{Z}_{q}$.

Let us illustrate the Theorem 1 with the following example:

Example 1: Let us assume that $q=2, p=3, m=3, k=1$ and $s=2$. Let us take the GBF $f:\{0,1\}^{3} \rightarrow \mathbb{Z}_{2}$ as follows: $f=x_{1} x_{2}$, where $G\left(\left.f\right|_{x_{0}=0}\right)$ and $G\left(\left.f\right|_{x_{0}=1}\right)$ give a path with $x_{2}$ as one of the end vertices. From (8), we have

$$
F^{\lambda}=x_{1} x_{2}+\frac{2 \lambda}{3}\left(x_{3}+2 x_{4}\right), G^{\lambda}=\bar{x}_{1} \bar{x}_{2}+\frac{2 \lambda}{3}\left(x_{3}+2 x_{4}\right),
$$

where $\lambda=0,1,2$. From (9) and (10), we have

$$
\begin{aligned}
U_{t}^{\lambda} & =\left\{F^{\lambda}+d_{0} x_{0}+t_{0} x_{0}+d x_{2}: d_{0}, d \in\{0,1\}\right\} \\
V_{t}^{\lambda} & =\left\{G^{\lambda}+d_{0} \bar{x}_{0}+t_{0} \bar{x}_{0}+\bar{d} x_{2}: d_{0}, d \in\{0,1\}\right\},
\end{aligned}
$$

where $\left(t_{0}\right)$ is the binary vector representation of $t$. Therefore, $\left\{\psi_{8}\left(U_{t}^{\lambda}\right), \psi_{8}^{*}\left(V_{t}^{\lambda}\right): 0 \leq t \leq 1,0 \leq \lambda \leq 2\right\}$ forms $(12,8)$ $\mathrm{ZCCS}_{4}^{24}$ which also optimal. The components of each code word from a code in $(12,8)$-ZCCS 44 are drawn from the roots of the polynomial: $z^{\delta}-1$, where $\delta=l c m(p, q)=l c m(2,3)=6$.

Remark 2: From (14) and (15), we see that $\psi_{2^{m+s}-p 2^{m}}\left(U_{t}^{\lambda}\right)$ and $\psi_{2^{m+s}-p 2^{m}}^{*}\left(V_{t}^{\lambda}\right)$ can also be expressed as the concatenation of $\omega_{p}^{\lambda(i-1)} \psi\left(C_{t}\right)$ and $\omega_{p}^{-\lambda(i-1)} \psi^{*}\left(\bar{C}_{t}\right)$, respectively, where $i=1,2, \ldots, p$. Therefore, the proposed PBF generators establish a link between the proposed direct construction and the indirect constructions of ZCCSs which are obtained by performing cocatenation operation on the CCCs from [4].

\section{Conclusions}

In this paper, we have developed a direct construction of optimal ZCCS with NPT lengths. Unlike the current state-ofthe-art which can only generate sub-optimal ZCCSs with NPT lengths, the novelty of this work stems from the use of PBFs.

\section{REFERENCES}

[1] H.-H. Chen, The Next Generation CDMA Technologies. Wiley, 2007.

[2] D. Carey, D. Roviras, and B. Senadji, "Comparison of multiple access interference in asynchronous MC-CDMA and DS-CDMA systems," in Proceedings Seventh International Symposium on Signal Processing and Its Applications., vol. 2, 2003, pp. 351-354. 
[3] P. Nagaradjane, A. Swaminathan, K. S. Dhyaneshwaran, B. R. Narayanasamy, and A. Ramakrishnan, "Multipath interference mitigation technique for MC DS/CDMA systems," in International Conference on Control, Automation, Communication and Energy Conservation, 2009, pp. 1-3.

[4] A. Rathinakumar and A. K. Chaturvedi, "Complete mutually orthogonal Golay complementary sets from Reed-Muller codes," IEEE Trans. Inf. Theory, vol. 54, no. 3, pp. 1339-1346, Mar. 2008.

[5] P. Sarkar, S. Majhi, and Z. Liu, "Optimal Z -complementary code set from generalized Reed-Muller codes," IEEE Trans. Commun, vol. 67, no. 3, pp. 1783-1796, Mar. 2019.

[6] P. Fan, W. Yuan, and Y. Tu, "Z-complementary binary sequences," IEEE Signal Process. Lett., vol. 14, no. 8, pp. 509-512, Aug. 2007.

[7] M. Golay, "Complementary series," IRE Trans. Inf. Theory, vol. 7, no. 2, pp. 82-87, Apr. 1961

[8] J. A. Davis and J. Jedwab, "Peak-to-mean power control in OFDM, Golay complementary sequences, and Reed-Muller codes," IEEE Trans. Inf. Theory, vol. 45, no. 7, pp. 2397-2417, Nov. 1999.

[9] C. Chen, "A novel construction of Z-complementary pairs based on generalized Boolean functions," IEEE Signal Process. Lett., vol. 24, no. 7, pp. 987-990, July 2017.

[10] C. Pai, S. Wu, and C. Chen, "Z-complementary pairs with flexible lengths from generalized Boolean functions," IEEE Commun. Lett., vol. 24 , no. 6, pp. 1183-1187, 2020.

[11] A. R. Adhikary, P. Sarkar, and S. Majhi, "A direct construction of $q$-ary even length Z-complementary pairs using generalized Boolean functions," IEEE Signal Process. Lett., vol. 27, pp. 146-150, 2020.

[12] L. Feng, P. Fan, X. Tang, and K. K. Loo, "Generalized pairwise Zcomplementary codes,” IEEE Signal Process. Lett., vol. 15, pp. 377-380, 2008.

[13] Z. Liu, Y. L. Guan, B. C. Ng, and H.-H. Chen, "Correlation and set size bounds of complementary sequences with low correlation zone," IEEE Trans. Commun., vol. 59, no. 12, pp. 3285-3289, Dec. 2011.

[14] K. G. Paterson, "Generalized Reed-Muller codes and power control in OFDM modulation," IEEE Trans. Inf. Theory, vol. 46, no. 1, pp. 104 120, Jan. 2000.

[15] P. Sarkar, S. Majhi, and Z. Liu, "A direct and generalized construction of polyphase complementary set with low PMEPR," in 2019 IEEE International Symposium on Information Theory (ISIT), 2019, pp. 22792283.

[16] - "A direct and generalized construction of polyphase complementary sets with low PMEPR and high code-rate for OFDM system," IEEE Trans. Commun., 2020.

[17] S. Das, S. Budišin, S. Majhi, Z. Liu, and Y. L. Guan, "A multiplier-free generator for polyphase complete complementary codes," IEEE Trans. Signal Process., vol. 66, no. 5, pp. 1184-1196, Mar. 2018.

[18] Z. Liu, Y. L. Guan, and U. Parampalli, "New complete complementary codes for peak-to-mean power control in multi-carrier CDMA," IEEE Trans. Commun., vol. 62, no. 3, pp. 1105-1113, Mar. 2014.

[19] Z. Liu, Y. L. Guan, and H.-H. Chen, "Fractional-delay-resilient receiver design for interference-free MC-CDMA communications based on complete complementary codes," IEEE Trans. Wireless Commun., vol. 14, no. 3, pp. 1226-1236, Mar. 2015.

[20] S. Das, S. Majhi, and Z. Liu, "A novel class of complete complementary codes and their applications for apu matrices," IEEE Signal Process. Lett., vol. 25, no. 9, pp. 1300-1304, Sept. 2018.

[21] P. Sarkar and S. Majhi, "A direct construction of optimal zccs with maximum column sequence PMEPR two for MC-CDMA system," IEEE Commun. Lett., 2020.

[22] S. W. Wu, A. Şahin, Z. M. Huang, and C. Y. Chen, "Z-complementary code sets with flexible lengths from generalized Boolean functions," IEEE Access, vol. 9, pp. 4642-4652, 2021.

[23] S. Wu and C. Chen, "Optimal Z-complementary sequence sets with good peak-to-average power-ratio property," IEEE Signal Process. Lett., vol. 25, no. 10, pp. 1500-1504, Oct. 2018.

[24] P. Sarkar, A. Roy, and S. Majhi, "Construction of Z-complementary code sets with non-power-of-two lengths based on generalized Boolean functions," IEEE Commun. Lett., pp. 1-5, 2020.

[25] S. Das, U. Parampalli, S. Majhi, Z. Liu, and S. Budišin, "New optimal Z-complementary code sets based on generalized paraunitary matrices," IEEE Trans. Signal Process., vol. 68, pp. 5546-5558, 2020.

[26] A. Adhikary and S. Majhi, "New construction of optimal aperiodic Zcomplementary sequence sets of odd-lengths," Electron. Lett., vol. 55, no. 19, pp. 1043-1045, 2019

[27] J. Li, A. Huang, M. Guizani, and H.-H. Chen, "Inter group complementary codes for interference resistant CDMA wireless communications," IEEE Trans. Wireless Commun., vol. 7, no. 1, pp. 166-174, Jan. 2008.
[28] W. Yuan, Y. Tu, and P. Fan, "Optimal training sequences for cyclicprefix-based single-carrier multi-antenna systems with space-time blockcoding," IEEE Trans. Wireless Commun., vol. 7, no. 11, pp. 4047-4050, Nov. 2008.

[29] H. M. Wang, X. Q. Gao, B. Jiang, X. H. You, and W. Hong, "Efficient MIMO channel estimation using complementary sequences," IET Commun., vol. 1, no. 5, pp. 962-969, Oct. 2007.

[30] V. K. Leont'ev, "On pseudo-boolean polynomials," Comput. Math. and Math. Phys., vol. 55, pp. 1926-1932, 2015.

[31] P. P. Vaidyanathan, "Ramanujan sums in the context of signal processingPart I: Fundamentals," IEEE Trans. Signal Process., vol. 62, no. 16, pp. 4145-4157, 2014. 